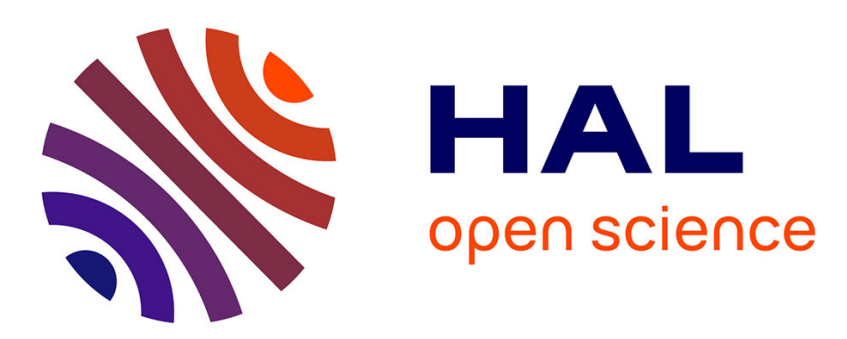

\title{
Femtosecond Two-Photon Absorption Laser Induced Fluorescence (fs-TALIF) Imaging of Atomic Nitrogen in Nanosecond Repetitive Discharges
}

Ciprian Dumitrache, Arnaud Gallant, Gabi Daniel Stancu, Christophe O. Laux

\section{To cite this version:}

Ciprian Dumitrache, Arnaud Gallant, Gabi Daniel Stancu, Christophe O. Laux. Femtosecond TwoPhoton Absorption Laser Induced Fluorescence (fs-TALIF) Imaging of Atomic Nitrogen in Nanosecond Repetitive Discharges. AIAA Scitech 2019 Forum, 2019, San Diego, France. 10.2514/6.2019-1507 . hal-02494206

\author{
HAL Id: hal-02494206 \\ https://hal.science/hal-02494206
}

Submitted on 28 Feb 2020

HAL is a multi-disciplinary open access archive for the deposit and dissemination of scientific research documents, whether they are published or not. The documents may come from teaching and research institutions in France or abroad, or from public or private research centers.
L'archive ouverte pluridisciplinaire HAL, est destinée au dépôt et à la diffusion de documents scientifiques de niveau recherche, publiés ou non, émanant des établissements d'enseignement et de recherche français ou étrangers, des laboratoires publics ou privés. 


\title{
Femtosecond Two-Photon Absorption Laser Induced Fluorescence (fs-TALIF) Imaging of Atomic Nitrogen in Nanosecond Repetitive Discharges
}

\author{
Ciprian Dumitrache ${ }^{1}$, Arnaud Gallant ${ }^{2}$, Gabi-Daniel Stancu ${ }^{3}$, Christophe Laux ${ }^{4}$ \\ Laboratoire EM2C, CNRS, CentraleSupélec, Université Paris-Saclay, 91190 Gif-sur-Yvette, France
}

\begin{abstract}
Presented herein is a feasibility study for absolute measurements of ground state atomic nitrogen inside of a nanosecond repetitive pulse (NRP) discharge using a femtosecond twophoton absorption laser induced fluorescence (TALIF) technique. A two-photon absorption scheme $(\lambda=2 \times 206.6 \mathrm{~nm})$ is used to probe the ground state population of atomic nitrogen $\left(2 \mathrm{p}^{3}\right.$ $\left.{ }^{4} \mathrm{~S}\right)$ inside the discharge. The scheme involves the excitation of the $3 p{ }^{4} \mathrm{~S}_{3 / 2}$ level with the subsequent fluorescence collection taking place from the radiative decay to the $3 \mathrm{~s}{ }^{4} \mathrm{P}$ triplet state around $745 \mathrm{~nm}$. The main challenge is to demonstrate the ability to perform the measurements at above atmospheric conditions where the typical ns-TALIF diagnostics is unable to provide information due to the high rate of collisional quenching. As such, the current investigation focuses on high-pressure discharges in pure nitrogen. We demonstrate here the ability to measure fs-TALIF signals from atomic nitrogen in NRP discharges operated at pressures between 0.1-5 bar. Additionally, we perform fs-TALIF measurements in Krypton and we identify the main fluorescence channels using the $\lambda=2 \times 204.13 \mathrm{~nm}$ excitation scheme. We report that significant saturation effects was observed in both mixtures due to the presence of photoionization which resulted in a departure from the $I^{2}$ dependency of the TALIF intensity.
\end{abstract}

\section{Introduction}

$\mathrm{E}$ fficient production of large amounts of nitrogen atoms is of importance for many industrial applications such as the enhancement of nitridation processes ${ }^{1}$, the synthesis of novel nanomaterials ${ }^{2}$ or biomedical treatments ${ }^{3,4}$. Moreover, it is also important to understand the high-pressure kinetic pathways that lead to atomic nitrogen production in plasma-assisted combustion in order to evaluate the plasma influence on NOx generation ${ }^{5,6}$. Despite the growing interest, measurements of the ground-state atomic nitrogen are challenging to perform because the excitation photon energy lies in the VUV range $(\lambda=100-120 \mathrm{~nm})$ which is not readily accessible by direct absorption spectroscopy. The present contribution seeks to address an existing gap in the literature by proposing measurements of the groundstate population of atomic nitrogen in a nanosecond repetitive pulsed (NRP) discharge at pressures above 1 bar using fs-TALIF as a diagnostic tool.

The first nitrogen TALIF experiment was performed by Bischel et al. ${ }^{7}$ inside a flow discharge in which atomic nitrogen was obtained by $\mathrm{N}_{2}$ dissociation in a $\mathrm{He}_{+} \mathrm{SF}_{6}$ buffer gas mixture at $\sim 10$ Torr. Their proposed scheme involved the two-photon excitation of the $3 \mathrm{p}{ }^{4} \mathrm{D}$ multiplet state at $\lambda=2 \times 211 \mathrm{~nm}$ with fluorescence collection taking place in the NIR at $868 \mathrm{~nm}$ following the radiative decay: $3 \mathrm{p}{ }^{4} \mathrm{D} \leftarrow 3 \mathrm{~s}{ }^{4} \mathrm{P}_{5 / 2}$. In our experiments, we are using a different TALIF excitation scheme involving the $3 \mathrm{p}^{4} \mathrm{~S}_{3 / 2}$ level at $\lambda=2 \times 206.6 \mathrm{~nm}$. This scheme was first proposed by Adams et al. and it has been shown in the past to present some distinct advantages compared to the scheme proposed by Bischel. Despite the higher photon energy, the $3 \mathrm{p}^{4} \mathrm{~S}_{3 / 2}$ state benefits from a lower quenching rate of the upper state by $\mathrm{N}_{2}{ }^{8}$. Moreover, the collection of fluorescence takes place in the visible spectrum $(\lambda=745 \mathrm{~nm})$ where the quantum efficiency of the most detectors is notably higher. Es-sebbar et al. have used optical emission spectroscopy together with the same N-TALIF scheme proposed here to characterize the post-discharge of a flowing microwave plasma source

\footnotetext{
${ }^{1}$ Postdoctoral Fellow, ciprian.dumitrache@centralesupelec.fr, AIAA Member

${ }^{2}$ PhD Student, arnaud.gallant@centralesupelec.fr

${ }^{3}$ Professor, gabi-daniel.stancu@ centralesupelec.fr

${ }^{4}$ Professor, chirstophe.laux@centralesupelec.fr, AIAA Associate Fellow
} 
operating at $2.45 \mathrm{GHz}$. They were able to measure a maximum $\mathrm{N}\left({ }^{4} \mathrm{~S}\right)$ density of $n_{e}=2.2 \times 10^{15} \mathrm{~cm}^{-3}$ for a pressure of 22 Torr using a nanosecond laser system for excitation ${ }^{9}$.

It has been shown in the past that ns-TALIF is a reliable technique for measurements below several tens of milibars ${ }^{10}$. Above this pressure, the quenching of the excited state becomes the major loss mechanism with a time scale even faster than the laser pulse length, i.e. below ns at atmospheric pressure ${ }^{11}$. Therefore, the main challenge of this study is to develop a "quenching free" diagnostic technique in the pressure range of interest ( $\mathrm{p}=1-10 \mathrm{bar}$ ) using a femtosecond laser as the excitation source. Previous experimental work performed by Kulatilaka et al. demonstrated fs-TALIF for $\mathrm{H}$-atom density measurements in a $\mathrm{CH}_{4} /$ Air Bunsen flame at atmospheric conditions ${ }^{12}$. In their system, the laser excitation wavelength $(\lambda=2 \times 205 \mathrm{~nm})$ is obtained by pumping an optical parametric amplifier (OPA) with a Ti:Sapphire femtosecond laser. Two-dimensional measurements of atomic oxygen densities inside an NRP discharge are demonstrated for the first time by Schmidt et al. ${ }^{13}$. They report local number densities ranging from $10^{13}-$ $10^{16} \mathrm{~cm}^{-3}$ for atomic oxygen with high spatial resolution $(\sim 15 \mu \mathrm{m})$ and fast acquisition rate $(\sim 1 \mathrm{kHz})$.

The current study reports on the development of a fs-TALIF technique for performing density measurements for atomic nitrogen inside of an NRP discharge using the $\lambda=2 \times 206.6 \mathrm{~nm}$ excitation scheme. We report that fluorescence signal was successfully collected inside the discharge in a pressure range corresponding to $\mathrm{p}=0.1-5$ bar. This demonstrates the ability of our technique to perform measurements in conditions that are heavily affected by collisional quenching in nanosecond regime. Additionally, measurements inside of a well-known quantity of krypton were also carried-out using a $\lambda=2 \times 204.1 \mathrm{~nm}$ scheme. The purpose of the krypton measurement is to provide a method of calibration for the determination of absolute atomic nitrogen densities in future studies. We report that the signal is affected by saturation due to the presence of photoionization in both gas mixtures.

Section II discusses the theory behind two-photon absorption laser induced fluorescence in two cases: low excitation limit (unsaturated regime) and high fluence (or saturated regime). The experimental setup used to perform fs-TALIF measurements in atomic nitrogen and krypton is presented in Section III and the experimental results are presented in Section IV. Finally, the manuscript concludes with conclusions and plans for future work in Section V.

\section{Theory}

The objective of this section is to provide a description of the calibration method used for TALIF diagnostics to determine ground-state densities of atomic nitrogen in an NRP discharge. The proposed method is based on a comparative measurement at a spectrally close two-photon resonance of krypton gas ${ }^{14}$. The transitions responsible for fluorescence emission are described in Fig. 1 below for both atomic nitrogen and krypton. Briefly, the krypton excitation scheme involves the absorption of two photons at $204.13 \mathrm{~nm}$ from the ground-state $4 \mathrm{p}{ }^{1} \mathrm{~S}$ to the first excited state $5 p^{\prime}[3 / 2]_{2}$. Fluorescence is emitted at $826.3 \mathrm{~nm}$ during the radiative decay: $5 \mathrm{p}^{\prime}[3 / 2]_{2} \rightarrow 5 \mathrm{~s}^{\prime}[1 / 2]_{1}$. The motivation for using $\mathrm{Kr}$ as a calibration source stems from the fact that the excitation scheme is at a similar wavelength with the desired atomic nitrogen scheme. This enables us to use the same laser system for both the $\mathrm{Kr}$ calibration and the Natom TALIF experiment. In addition, the noble gas is found naturally in its atomic ground-state (unlike the atomic nitrogen which needs to be dissociated first) and can be metered with precision inside the reactor for the calibration experiment.

The calibration itself is done by filling the reactor with a known concentration of $\mathrm{Kr}$ and measuring the fluorescence signal intensity at this known condition. Several prerequisites are necessary in order for the calibration to be valid. Firstly, both measurements need to be performed with the same spatial, spectral and temporal laser intensity distribution. This requirement eliminates the need to completely characterize the laser radiation source. Secondly, it is necessary that the fluorescence is collected in the non-saturated regime (i.e. the fluorescence signal

scales $\sim I_{\text {laser }}{ }^{2}$ ). Otherwise, the effects of resonance-induced photoionization (REMPI) and their respective crosssections need to be known a priori. In the case of low photon fluxes, the ground-state atomic densities remain almost the same and the integrated fluorescence signal for species " $\mathrm{x}$ ", is given by ${ }^{15}$ :

$$
S_{T A L I F}^{X}=n_{X} A l \frac{\Omega}{4 \pi} T \eta_{Q E} W_{2 p h} a_{X}
$$




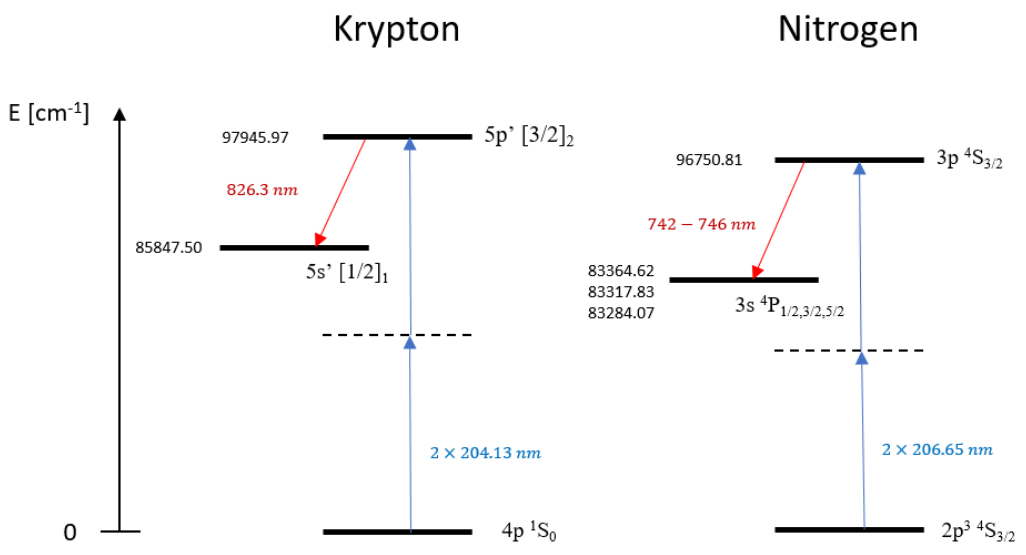

Figure 1: Energy Level Diagram for $\mathrm{Kr}$ and $\mathrm{N}$-atom TALIF

Where $n_{X}$ represents the number density of species "x", $A l$ is the signal collection volume, $\frac{\Omega}{4 \pi}$ is the collection solid angle, $\mathrm{T}$ is the transmission of the optical system (windows, lenses, mirrors) and $\eta_{Q E}$ is the quantum efficiency of the detector. The two-photon excitation rate, $W_{2 p h}$, is expressed in terms of a two-photon cross section, $\sigma^{(2)}$ which has been determined experimentally in the past for ns-TALIF:

$$
W_{2 p h}=G^{(2)} \sigma^{(2)} g(\Delta v)\left(\frac{I}{h v}\right)^{2}
$$

In the equation above, $G^{(2)}$ is the photon statistic factor (typically taken $\sim 2^{16,17}$ ), $g(\Delta v)$, is the normalized line profile $\left(\int_{-\infty}^{+\infty} g(v) d v=1\right)$ which comprises of a convolution between the laser photon spectral profile and the absorption line of the atom as described by Niemi et al. ${ }^{10}$. It is important to note that, since the TALIF scheme involves the absorption of two photons, the collected signal is proportional to the square of the laser intensity. Thus, the TALIF signal depends strongly on the laser beam quality (i.e. ability to tightly focus a beam) and the laser pulse duration. The last quantity in Eq. (1) represents the optical branching ratio of the observed fluorescence transition and is given by:

$$
a_{X}=\frac{A_{2 i}}{\sum_{i} A_{2 i}+Q}
$$

From a physical point of view, the optical branching ratio is a measure of the efficiency with which fluorescence signal is being generated from the excited state populated by two-photon absorption (denoted here with subscript 2 ). It is the ratio between the spontaneous emission over channel " $\mathrm{i}$ " of the multiplet state, $\mathrm{A}_{2 \mathrm{i}}$, and the effective de-excitation rate $\sum_{i} A_{2 i}+Q$, where $\mathrm{Q}$ is the collisional quenching rate.

Finally, the absolute nitrogen density is obtained by dividing the signal obtained experimentally in the discharge (according to Eq. 1) by the TALIF signal in pure Kr:

$$
n_{N}=n_{K r} \frac{S^{N}{ }_{T A L I F}}{S^{K r}{ }_{T A L I F}} \chi
$$

The factor $\chi$ represents the ratio of atomic and experimental constants and is determined partly experimentally and partly from literature (especially the ratios of quenching rates and excitation cross-sections).

However, it is important to note that Eq. (1) above is only valid under the assumption of low excitation (i.e. far from saturation) when the ground level is not significantly depopulated. In the case of high laser intensity (such as in the case of femtosecond laser pulse excitation), this assumption is not necessarily valid. For example, photo-ionization by a $2+1$ REMPI, in which the state $3 p{ }^{4} S_{3 / 2}$ acts as the intermediate excitation state, could be significant. If REMPI is to be included into the model, Eq. 1 becomes: 


$$
S^{X}{ }_{T A L I F}=h v A l T \eta_{Q E} \frac{\Omega}{4 \pi}\left[\frac{W_{2 p h} A_{2 i}}{W_{R E M P I}+\sum_{i} A_{2 i}+Q}\right]\left[1-\exp \left(-\left\{W_{R E M P I}+\sum_{i} A_{2 i}+Q\right\} t\right)\right]
$$

Additionally, strongly pumped excited states could become much more populated than the nearby electronic states which will result in population inversion and stimulated emission between these levels. When the stimulated emission is present, it reduces the population of the excited level and acts to further reduce the measured TALIF signal ${ }^{15,18}$. In this particular scenario, the calibration becomes cumbersome as the REMPI cross-sections and the stimulated emission need to be determined for both mixtures in order to perform the calibration.

A few important differences between fs-TALIF and ns-TALIF techniques should be noted. The two-photon excitation rate in case of fs-lasers is lowered due to the broad laser spectral profile and is largely increased because the laser is mode-locked, the photon statistic factor being much higher in case of fs lasers. A "quench-free" fs-TALIF technique can be implemented when lasers with high photon flux are employed. As shown in the work of Stancu ${ }^{19}$, the fluorescence signals become independent on quenching phenomena. However, Rabi oscillation and Stark shift effects need to be considered to correctly model the laser-atom interaction.

\section{Experimental Setup}

The optical layout used for the fs-TALIF experiment is presented in Fig. 1 below. The femtosecond laser pulses are produced by a Ti:Sapphire laser system (Spectra Physics Solstice ACE) which operates in the wavelength range $\lambda$ $=780-830 \mathrm{~nm}$. The actual laser system comprises of three components: a femtosecond seed laser (Spectra Physics MaiTai), a nanosecond Q-switched pump laser (Spectra Physics Empower) and a regenerative amplifier stage. The seed laser is a mode-locked Ti:Sapphire laser that produces femtosecond pulses $(\sim 100 \mathrm{fs})$ with low peak power at high repetition rate (average power $\sim 1$ Watt, at $1 \mathrm{MHz}$ ). The pump laser is a solid state Nd:YLF that operates at a wavelength of $527 \mathrm{~nm}$ generating pulses with a power in excess of 40 Watts and a repetition rate of $1 \mathrm{kHz}$. The operational principle of the laser is based on the concept of chirped pulse amplification: the fs pulse generated by the seed laser is stretched in time inside the chirped stretcher using a grating and it is passed through a Ti:Sapphire gain medium that has been previously excited by the pump beam of the Nd:YLF laser. At the exit of the amplifier cavity, the pulse is compressed again with another grating to recover the femtosecond pulse. In its current configuration, the Solstice Ace system provides $100 \mathrm{fs}$ pulses with an average pulse power $\sim 7$ Watts at a repetition rate of $1 \mathrm{kHz}$. The output beam of the Ti:Sapphire laser $(\lambda=828 \mathrm{~nm})$ is frequency quadrupled to reach the TALIF wavelengths using a third harmonic stage (Spectra Physcs TP-1A-THG) followed by a fourth harmonic stage (Spectra Physics TP-1AFHG). The third harmonic generation is obtained by means of two second order non-linear processes: second harmonic generation $\left(\omega_{1}+\omega_{1}=\omega_{2}\right)$ followed by sum frequency generation $\left(\omega_{1}+\omega_{2}=\omega_{3}\right)$. A timeplate is installed between the second and the third harmonic generation stages in order to eliminate the time delay caused by the group velocity dispersion (GVD) of the crystal and optics. Finally, the fourth harmonic light is obtained by mixing the third harmonic light with the fundamental $\left(\omega_{1}+\omega_{3}=\omega_{4}\right)$. The power obtained at the fourth harmonic as well as the leakage of the other harmonics at the output of the frequency quadrupling stage have been measured and are summarized in Table 1 below.

Table 1: Laser power for each of the harmonics measured at the output after a separating prism

\begin{tabular}{cccc}
\hline Harmonic & Wavelength [nm] & Power [mW] & Polarization \\
\hline $1^{\text {st }}$ & 828 & 4 & Vertical \\
$2^{\text {nd }}$ & 414 & 3.5 & Vertical \\
$3^{\text {rd }}$ & 276 & 2 & Vertical \\
$4^{\text {th }}$ & 207 & 62 & Horizontal
\end{tabular}

The fourth harmonic is selected at the laser exit using a pair of dichroic mirrors and a prism which has the role separating the $4^{\text {th }}$ harmonic that will be used for the TALIF experiment from the leakage light from the other harmonics (in particular, the fundamental which is spectrally overlapped with the krypton fluorescence wavelength at $826 \mathrm{~nm}$ and needs to be suppressed). The TALIF beam is focused inside of the chamber using a $\mathrm{f}=125 \mathrm{~mm}$ lens to generate beam waist of $50 \mu \mathrm{m}$. The spot size was measured by traversing the beam with a razor blade according to the method 
described by Siegman ${ }^{20}$. A beam quality corresponding to $\mathrm{M}^{2}=6$ was obtained by fitting the beam radius along the Rayleigh range (See Fig. 2) according to the formula:

$$
w^{2}(z)=w_{0}{ }^{2}+\frac{\lambda}{\pi w_{0}{ }^{2}} M^{2}\left(z-z_{0}\right)^{2}
$$

Finally, the fluorescence signal is collected using an $\mathrm{f} / 2.8$ lens and imaged onto the entrance slit of spectrometer (Acton SP-500i). The entrance slit width was set to 500 microns and the entire slit height is imaged vertically onto the detector array of a ICCD camera (Princeton Instruments PiMax 2). For all the experiments reported here a 1200 grooves $/ \mathrm{mm}$ grating blazed at $500 \mathrm{~nm}$ was used. The dispersion was determined to be $0.034 \mathrm{~nm} / \mathrm{pixel}$, which yielded an on-ICCD spectral resolution of $0.43 \mathrm{~nm} / \mathrm{pixel}$ (pixel size corresponds to $12 \times 24 \mu \mathrm{m}$ for a $512 \times 512$ pixel array).

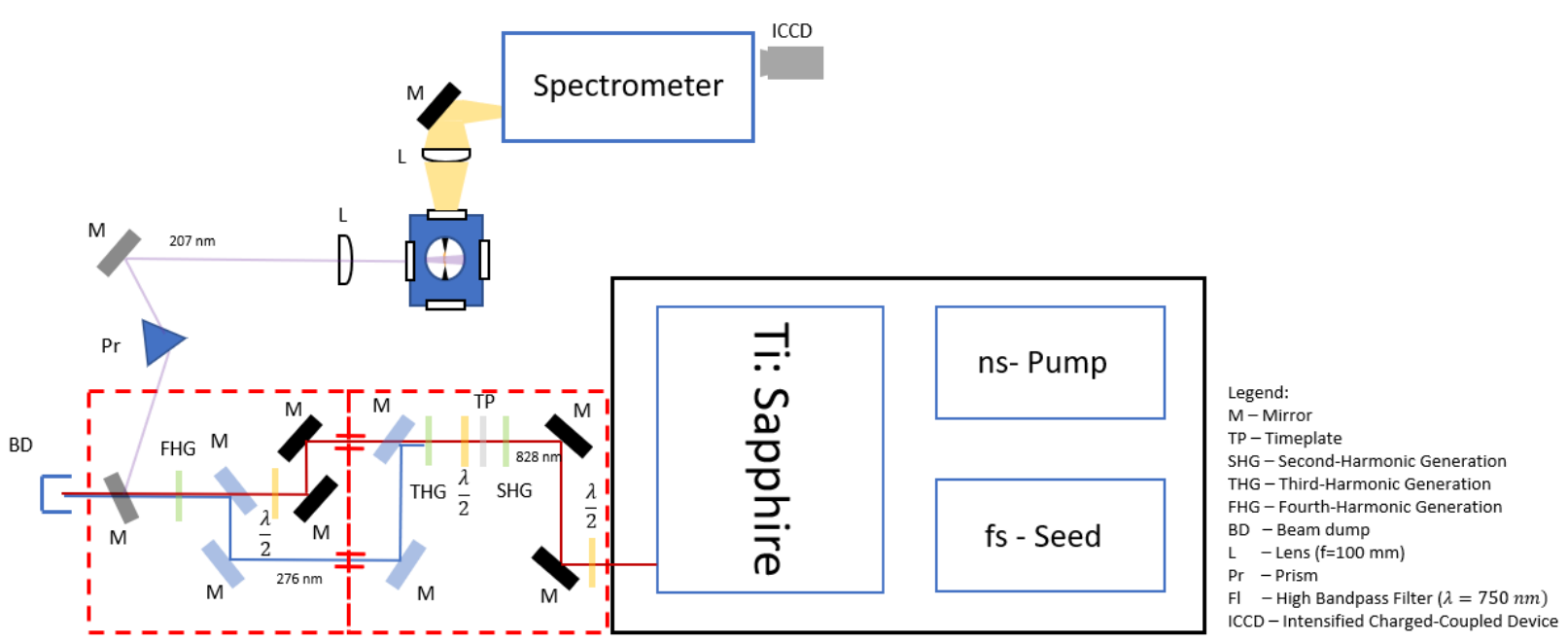

Figure 2: Optical Layout for the fs-TALIF experiments. The same setup is used for both $\mathrm{Kr}$ calibration and $\mathrm{N}$-atom measurements inside the NRP discharge

NRP discharges are produced with a FID FPG20-30MKS50 high-voltage pulse generator. The generator can deliver pulses of up to $20 \mathrm{kV}$, with a pulse length tunable between 10-50 ns at a maximum repetition rate of $30 \mathrm{kHz}$. The maximum current attained in NRP discharges is about $50 \mathrm{~A}$.

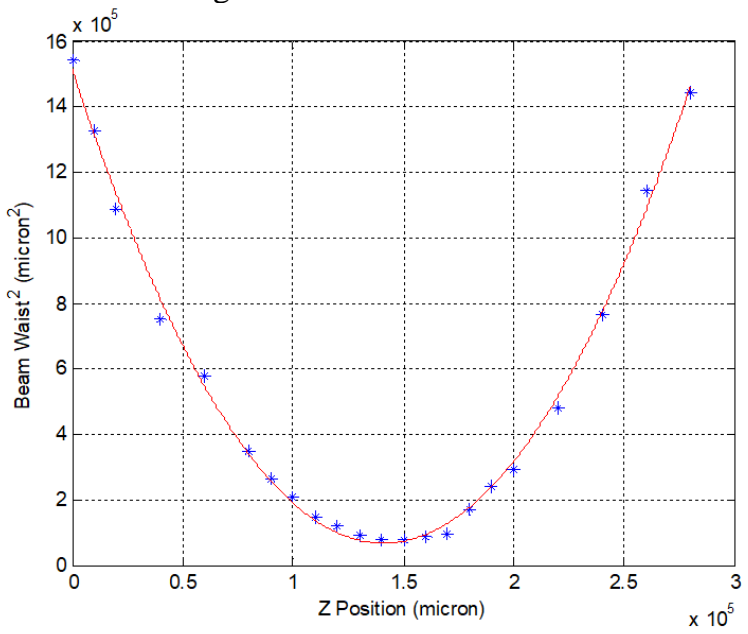

Figure 3: Measurements of the beam radius across the Rayleigh range. The fit allows one to determine the beam waist size $\left(\mathrm{w}_{0}=52 \mu \mathrm{m}\right)$ and beam quality $\left(\mathrm{M}^{2}=6\right)$.

American Institute of Aeronautics and Astronautics 


\section{Results and Discussions}

The two-photon absorption scheme in krypton generates emission over several channels as seen in Fig. 4. It is interesting to note that, due to the broad profile of the laser pulse (FWHM 0.4 nm), there are in fact 9 emission lines observed experimentally. Moreover, even though most of past literature have focused on the fluorescence emitted at $826.3 \mathrm{~nm}$ during the radiative decay: $5 \mathrm{p}^{\prime}[3 / 2]_{2} \rightarrow 5 \mathrm{~s}^{\prime}[1 / 2]_{1}$, the strongest emission was observed at $760 \mathrm{~nm}$ from the radiative decay of: $5 \mathrm{p}[3 / 2]_{2} \rightarrow 5 \mathrm{~s}[3 / 2]_{2}$. This transition is also more convenient to access due to the fact that it is not spectrally overlapped with the laser's fundamental frequency $(\lambda=818 \mathrm{~nm}, \mathrm{FWHM} \sim 10 \mathrm{~nm})$ and it is spectrally closer to the nitrogen triplet state that is probed in the NRP discharge which will alleviate some of the constraints on the detector quantum efficiency and transmission of optical system as seen in Eq. (1) and Eq. (5) respectively.

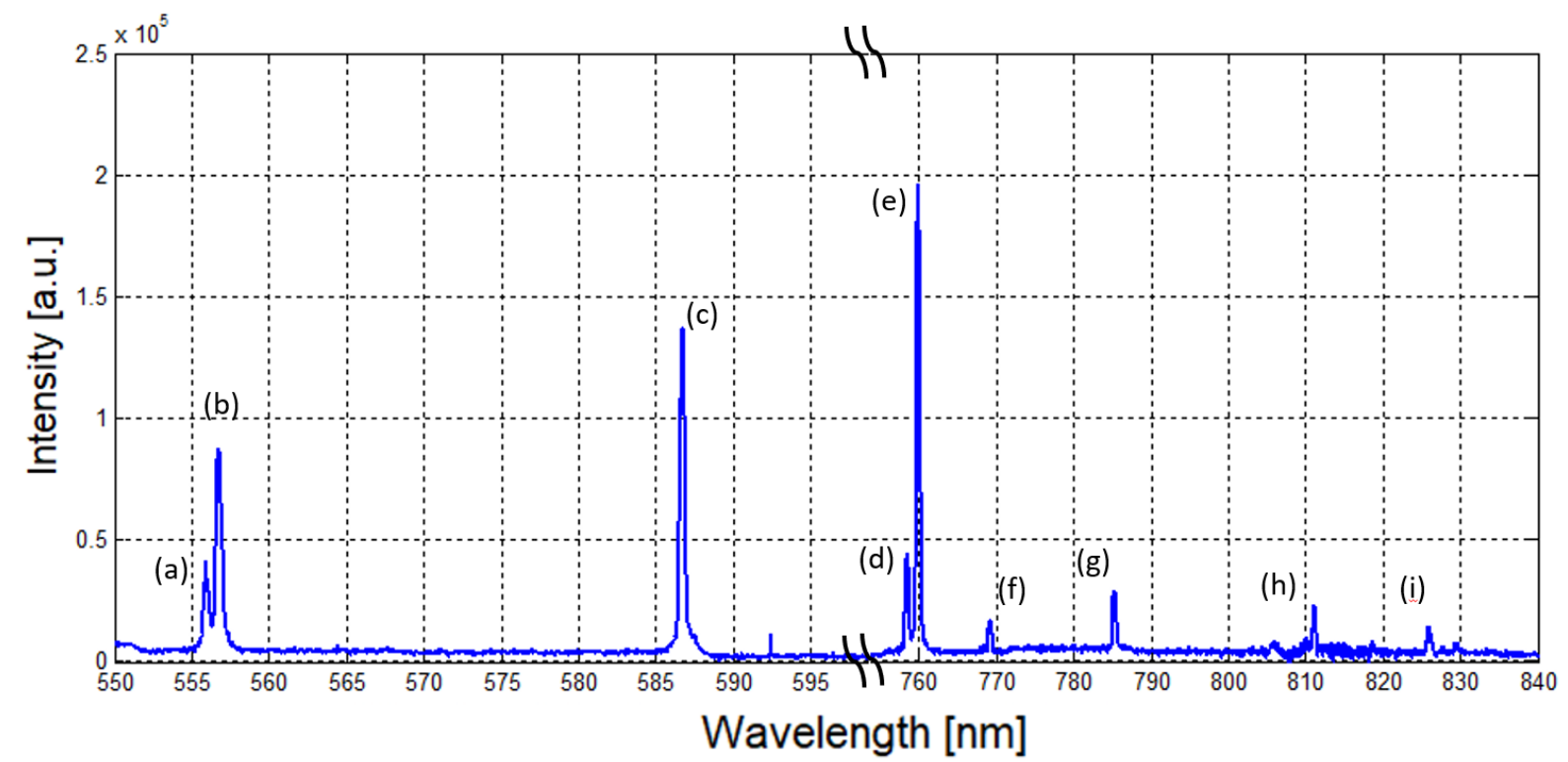

Figure 4: fs-TALIF emission spectra for Krypton. The fluorescence was obtained using the $\lambda=\mathbf{2} \times \mathbf{2 0 4 . 2} \mathbf{n m}$ excitation scheme.

Table 2: Spectroscopic data for the experimentally observed transitions in krypton (from NIST) ${ }^{21}$

\begin{tabular}{|c|c|c|c|c|c|}
\hline Line & Wavelength [nm] & Einstein Coefficient $\left[\mathrm{sec}^{-1}\right]$ & Lower Level, Term, J & & Upper Level, Term, J \\
\hline (a) & 556.22 & $1.10 \mathrm{E}+05$ & $4 s^{2} 4 p^{5}\left(2 \mathrm{P}_{3 / 2}^{\circ}\right) 5 s, \quad{ }^{2}[3 / 2]^{\circ}$, & 2 & $4 s^{2} 4 p^{5}\left({ }^{2} P_{1 / 2}^{\circ}\right) 5 p, \quad 2[3 / 2], \quad 2$ \\
\hline (b) & 557.03 & $9.80 e+05$ & $4 s^{2} 4 p^{5}\left({ }^{2} P_{3 / 2}^{\circ}\right) 5 s$ & 2 & $4 s^{2} 4 p^{5}\left({ }^{2} p^{\circ}{ }_{1 / 2}\right) 5 p, \quad{ }^{2}[1 / 2], \quad 1$ \\
\hline (c) & 587.09 & $7.1 \mathrm{e}+05$ & $4 s^{2} 4 p^{5}\left({ }^{2} p^{\circ}{ }_{3 / 2}\right) 5 s$ & 1 & $4 s^{2} 4 p^{5}\left({ }^{2} p^{\circ}{ }_{1 / 2}\right) 5 p, \quad 2[3 / 2], \quad 2$ \\
\hline (d) & 758.74 & $4.310 e+07$ & $4 s^{2} 4 p^{5}\left({ }^{2} p^{\circ}{ }_{3 / 2}\right) 5 s$ & 1 & $4 s^{2} 4 p^{5}\left({ }^{2} \mathrm{p}^{\circ}{ }_{3 / 2}\right) 5 p, \quad{ }^{2}[1 / 2], \quad 0$ \\
\hline (e) & 760.15 & $2.732 \mathrm{e}+07$ & $4 s^{2} 4 p^{5}\left({ }^{2} p_{3 / 2}^{\circ}\right) 5 s$ & 2 & $4 s^{2} 4 p^{5}\left({ }^{2} p_{3 / 2}^{\circ}\right) 5 p, \quad 2[3 / 2], \quad 2$ \\
\hline (f) & 769.45 & $4.27 e+06$ & $4 s^{2} 4 p^{5}\left(2 p^{\circ}{ }_{3 / 2}\right) 5 s$ & 2 & $4 s^{2} 4 p^{5}\left({ }^{2} p^{\circ}{ }_{3 / 2}\right) 5 p, \quad{ }^{2}[3 / 2], \quad 1$ \\
\hline (g) & 785.48 & $2.041 \mathrm{e}+07$ & $4 s^{2} 4 p^{5}\left({ }^{2} p_{1 / 2}^{\circ}\right) 5 s$ & 0 & $4 s^{2} 4 p^{5}\left({ }^{2} p^{\circ}{ }_{1 / 2}\right) 5 p, \quad 2[1 / 2], \quad 1$ \\
\hline (h) & 811.29 & $3.610 e+07$ & $4 s^{2} 4 p^{5}\left(2 p^{\circ}{ }_{3 / 2}\right) 5 s$ & 2 & $4 \mathrm{~s}^{2} 4 \mathrm{p}^{5}\left({ }^{2} \mathrm{p}^{\circ}{ }_{3 / 2}\right) 5 \mathrm{p}, \quad{ }^{2}[5 / 2]$, \\
\hline (i) & 826.32 & $3.416 e+07$ & $4 s^{2} 4 p^{5}\left(2 P_{1 / 2}^{\circ}\right) 5 s$ & 1 & $4 s^{2} 4 p^{5}\left({ }^{2} p^{\circ}{ }_{1 / 2}\right) 5 p, \quad 2[3 / 2], \quad 2$ \\
\hline
\end{tabular}

The fluorescence signal for the atomic nitrogen inside the NRP discharge is collected from the radiative decay of: $3 p{ }^{4} S_{3 / 2} \rightarrow 3 \mathrm{~s}{ }^{4} \mathrm{P}_{1 / 2,3 / 2,5 / 2}$ triplet. Shown in Figure 3 is the TALIF signal collected at $10 \mu \mathrm{s}$ after the discharge using the spectrometer. All three transitions are observed with the strongest one being the $3 \mathrm{p}{ }^{4} \mathrm{~S}_{3 / 2} \rightarrow 3 \mathrm{~s}{ }^{4} \mathrm{P}_{5 / 2}$ transition (also see Table 3 for the comparison with the NIST database). It is worth noting that the triplet was also naturally observed in the plasma between 1 us and 5 us after the discharge. Thus, for the early times, the plasma background needs to be subtracted in order to obtain the pure TALIF signal. 


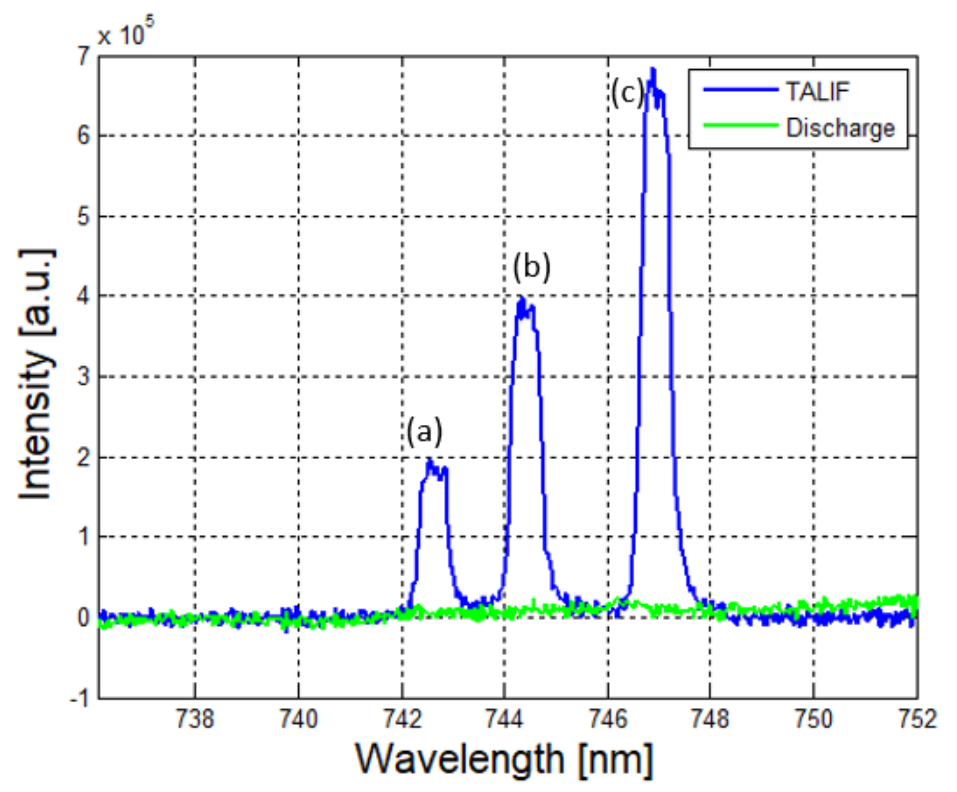

Figure 5: fs-TALIF emission spectra for atomic nitrogen inside of a NRP discharge. The fluorescence was obtained using the $\lambda=2 \times \mathbf{2 0 6 . 7} \mathbf{n m}$ excitation scheme and was collected at $10 \mu$ s after the discharge at $\mathrm{p}_{0}=100 \mathrm{mbar}$.

Table 3: Spectroscopic data for the experimentally observed transitions in atomic nitrogen (from NIST) ${ }^{21}$

\begin{tabular}{|c|c|c|c|c|}
\hline Line & Wavelength [nm] & Einstein Coefficient $\left[\mathrm{sec}^{-1}\right]$ & Lower Level, Term, J & Upper Level, Term, J \\
\hline (a) & 742.36 & $5.64 \mathrm{e}+06$ & $2 s^{2} 2 p^{2}(3 P) 3 s, \quad 4 P, 1 / 2$ & $2 s^{2} 2 p^{2}\left({ }^{3} P\right) 3 p,{ }^{4} S 0,3 / 2$ \\
\hline (b) & 744.23 & $1.19 e+07$ & $2 s^{2} 2 p^{2}\left({ }^{3} P\right) 3 s, \quad 4 P, 3 / 2$ & $2 s^{2} 2 p^{2}\left({ }^{3} P\right) 3 p,{ }^{4} S^{0}, 3 / 2$ \\
\hline (c) & 746.83 & $1.96 \mathrm{e}+07$ & $2 s^{2} 2 p^{2}\left({ }^{3} P\right) 3 s, \quad 4 P, 5 / 2$ & $2 s^{2} 2 p^{2}\left({ }^{3} P\right) 3 p,{ }^{4} S^{0}, 3 / 2$ \\
\hline
\end{tabular}

The krypton calibration method can be reliably used to obtain absolute densities of ground state atomic nitrogen in the discharge as long as the fluorescence signal is collected in the unsaturated regime. The influence of saturation on the TALIF signal is verified by plotting the fluorescence intensity as a function of laser fluence. According to Eq. (2), the TALIF signal is expected to vary with $I_{\text {laser }}^{2}$ if the effects of resonant multiphoton ionization and stimulated emission are negligible. From a physical point of view this means that there are no other population losses pathways from the two-photon excited state than spontaneous emission (fluorescence) and collisional quenching. However, a much lower dependency on laser intensity is observed experimentally for both krypton (Fig. 6-left) and atomic nitrogen (Fig. 6-right). This indicates the presence of additional loss channels such as 2+1 REMPI and (possibly) stimulated emission. To complement these observations, significant bremsstrahlung emission has been observed experimentally even after the plasma background has been subtracted which supports the idea that photoionization induced by the laser is present. It should be noted that, for a $100 \mathrm{fs}$ laser pulse, the spot size calculations presented in the previous section indicate a laser peak intensity on the order of $\sim 4 \mathrm{TW} / \mathrm{cm}^{2}$. This is much higher than the typical intensity required to achieve 2+1 REMPI even with nanosecond laser pulses ${ }^{22}$. In order to perform $\mathrm{N}$-atom TALIF measurements in the quench-free regime at elevated pressure one has to operate in conditions of high laser fluence which means that photoionization and stimulated emission needs to be considered. 

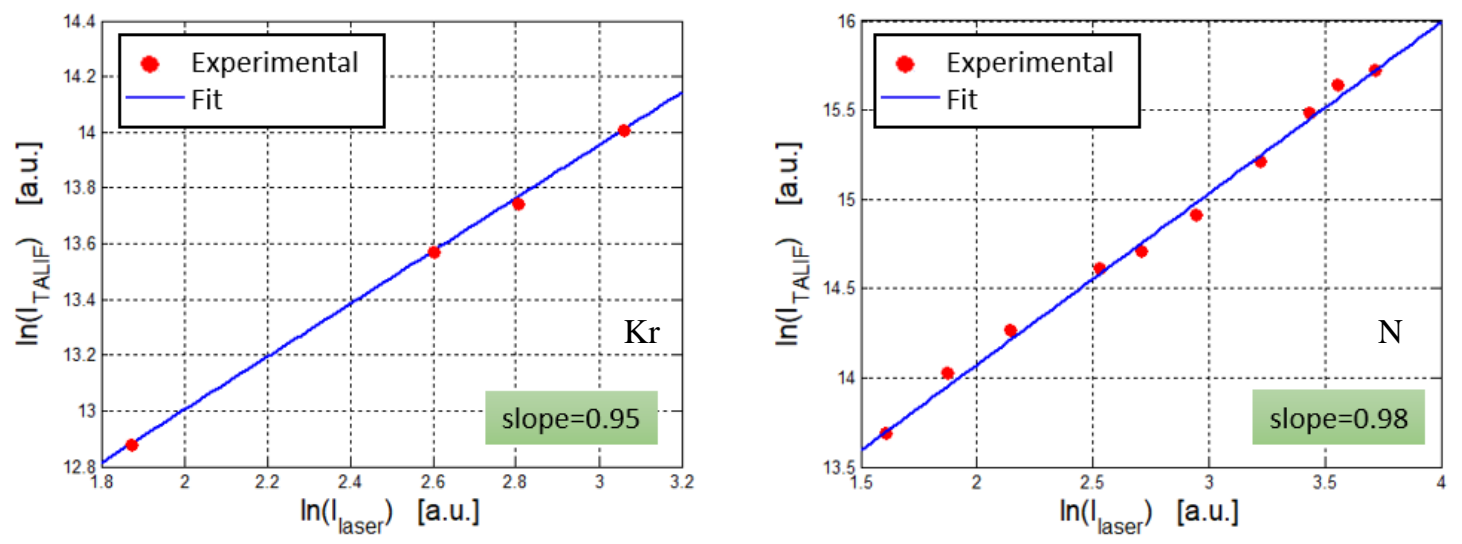

Figure 6: Fluorescence signal as a function of laser intensity for krypton (left) and atomic nitrogen (right). The experiments were conducted at $\mathrm{p}_{0}=100$ mbar.

The atomic nitrogen fluorescence signal decay after the discharge is shown at various pressures $(\mathrm{p}=0.1-2 \mathrm{bar})$ in Fig. 7 below. We note that initially the signal is stronger as we increase the pressure. This is due to the fact that there is more molecular nitrogen available to be dissociated in the discharge. However, the signal decays faster with time at higher pressure due to the faster three-body recombination of atomic nitrogen to form $\mathrm{N}_{2}(B)^{23-25}$. An interesting finding is that the TALIF signal stays relatively constant between 10-60 $\mu$ s (and even longer at low pressures). Over this range of time delays, the recombination is counter-balanced by the increase in ground state population through collisional de-excitation of atomic nitrogen excited states $\left(\mathrm{N}\left({ }^{2} \mathrm{P}\right), \mathrm{N}\left({ }^{2} \mathrm{D}\right)\right.$ and $\mathrm{N}\left({ }^{4} \mathrm{D}\right)$, in particular) ${ }^{23,26}$.

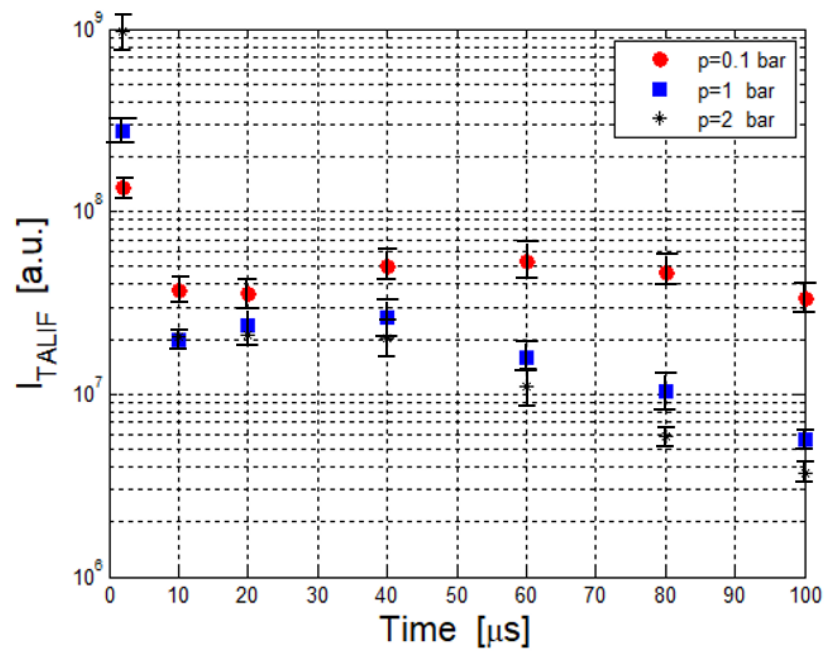

Figure 7: Atomic nitrogen TALIF signal evolution in time for various pressures inside of an NRP discharge $\left(\mathrm{V}_{\text {disch }}=8.5 \mathrm{kV}\right)$.

The highest pressure in which fs-TALIF signal was collected so far corresponds to 5 bar and was limited by the window thickness on our reactor and the ability to supply the voltage required to generate breakdown at higher pressures. As can be seen from Fig. 8, all three transitions of the triplet are still visible at 5 bars and the signal-to-noise level it's still reasonable high at 5 bars $\left(\mathrm{SNR}_{742 \mathrm{~nm}}=6.7, \mathrm{SNR}_{744 \mathrm{~nm}}=14, \mathrm{SNR}_{746 \mathrm{~nm}}=31.4\right)$ indicating that it is possible to go even further up in pressure once the chamber is adapted for higher pressure operation and the pulser power supply is augmented. 


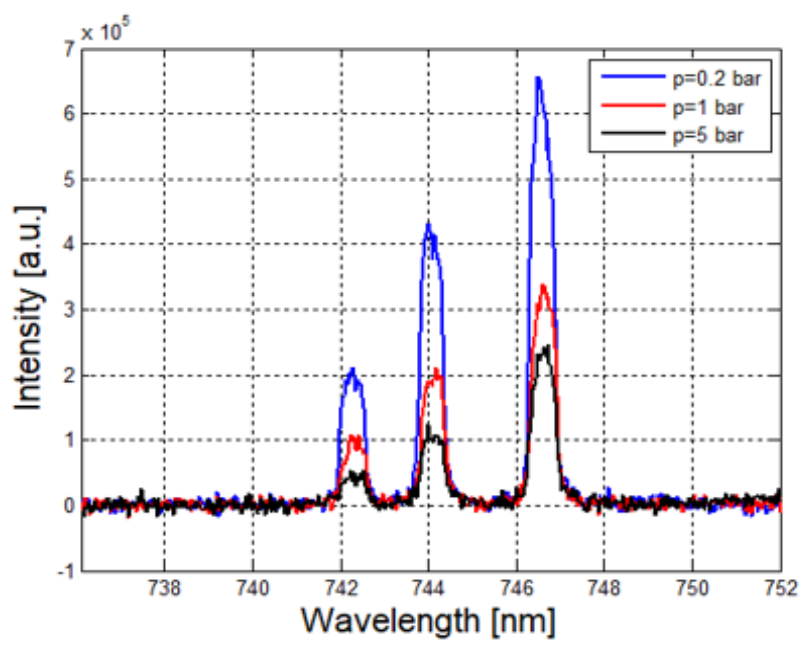

Figure 8: Fluorescence signal for atomic nitrogen at three different pressures. The signal was collected $10 \boldsymbol{\mu} \boldsymbol{s}$ after the discharge $\left(\mathrm{V}_{\text {disch }}=15 \mathrm{kV}\right)$.

\section{Conclusions and Future Work}

In this study, we performed measurements of the ground state atomic nitrogen $\left(2 \mathrm{p}^{3}{ }^{4} \mathrm{~S}\right)$ generated by dissociation of $\mathrm{N}_{2}$ in an NRP discharge at above-atmospheric pressures. To obtain fluorescence in this pressure range, a fs-TALIF experiment was developed. The fs-TALIF scheme developed here involves the excitation of the $3 \mathrm{p}^{4} \mathrm{~S}_{3 / 2}$ level $(\lambda=$ $2 \times 206.6 \mathrm{~nm}$ ) with the subsequent fluorescence collection taking place from the radiative decay to the $3 \mathrm{~s}{ }^{4} \mathrm{P}$ triplet state around $745 \mathrm{~nm}$. We are reporting that fluorescence signal was successfully collected for pressures as high as 5 bars with the ability to extend the range further in future studies. Additionally, we performed fs-TALIF measurements in krypton using a two-photon excitation scheme similar to the one used in nitrogen $(\lambda=2 \times 204.1 \mathrm{~nm})$. It is reported here that the fluorescence signal is affected by saturation in both gases due to the high laser fluence of the femtosecond laser system $\left(\mathrm{I}_{\text {laser }} \sim 4 \mathrm{TW} / \mathrm{cm}^{2}\right)$ which is different than previously reported in fs-TALIF literature. Future work will further develop the fs-TALIF technique presented herein in order to provide quantitative information for number density of the ground state atomic nitrogen using the krypton signal as a calibration source. Moreover, the technique will be expanded to provide temporally resolved 2-D spatial mappings of the atomic nitrogen inside the NRP discharge.

\section{References}

1 Cho, C., Kinemuchi, Y., Suematsu, H., Jiang, W., and Yatsui, K., "Enhancement of nitridation in synthesis of aluminum nitride nanosize powders by pulsed wire discharge," Japanese Journal of Applied Physics, Part 1: Regular Papers and Short Notes and Review Papers, vol. 42, 2003, pp. 1763-1765.

2 Pai, D. Z., Ostrikov, K. K., Kumar, S., Lacoste, D. A., Levchenko, I., and Laux, C. O., "Energy efficiency in nanoscale synthesis using nanosecond plasmas," Scientific Reports, vol. 3, 2013, pp. 1-7.

3 Noli, F., Pichon, L., and Öztürk, O., "The Influence of Plasma-Based Nitriding and Oxidizing Treatments on the Mechanical and Corrosion Properties of CoCrMo Biomedical Alloy," Metallurgical and Materials Transactions A: Physical Metallurgy and Materials Science, vol. 49, 2018, pp. 1383-1396.

Liu, R., Li, X., Hu, X., and Dong, H., "Surface modification of a medical grade Co-Cr-Mo alloy by lowtemperature plasma surface alloying with nitrogen and carbon," Surface and Coatings Technology, vol. 232, 2013, pp. 906-911.

5 Uddi, M., Jiang, N., Adamovich, I. V., and Lempert, W. R., "Nitric oxide density measurements in air and air/fuel nanosecond pulse discharges by laser induced fluorescence," Journal of Physics D: Applied Physics, vol. 42, 2009. 
Sun, W., and Ju, Y., "Nonequilibrium Plasma-Assisted Combustion: A Review of Recent Progress," J. Plasma Fusion Res., vol. 89, 2013, pp. 208-219.

Bischel, W. K., Perry, B. E., and Crosley, D. R., "Two-photon laser-induced fluorescence in oxygen and nitrogen atoms," Chemical Physics Letters, vol. 82, 1981, pp. 85-88.

Adams, S. F., and Miller, T. A., "Two-photon absorption laser-induced fluorescence of atomic nitrogen by an alternative excitation scheme," Chemical Physics Letters, vol. 295, 1998, pp. 305-311.

Es-Sebbar, E., Benilan, Y., Jolly, A., and Gazeau, M. C., "Characterization of an N2 flowing microwave postdischarge by OES spectroscopy and determination of absolute ground-state nitrogen atom densities by TALIF," Journal of Physics D: Applied Physics, vol. 42, 2009.

Dobele, H. F., Mosbach, T., Niemi, K., and Schulz-Von Der Gathen, V., "Laser-induced fluorescence measurements of absolute atomic densities: Concepts and limitations," Plasma Sources Science and Technology, vol. 14, 2005.

Agrup, S., Ossler, F., and Aldén, M., "Measurements of collisional quenching of hydrogen atoms in an atmospheric-pressure hydrogen oxygen flame by picosecond laser-induced fluorescence," Applied Physics B Lasers and Optics, vol. 61, 1995, pp. 479-487.

Kulatilaka, W. D., Gord, J. R., and Roy, S., "Femtosecond two-photon LIF imaging of atomic species using a frequency-quadrupled Ti: Sapphire laser," Applied Physics B: Lasers and Optics, vol. 116, 2014, pp. 7-13.

Schmidt, J. B., Roy, S., Kulatilaka, W. D., Shkurenkov, I., Adamovich, I. V, Lempert, W. R., and Gord, J. R., "Femtosecond, two-photon-absorption, laser-induced-fluorescence (fs-TALIF) imaging of atomic hydrogen and oxygen in non-equilibrium plasmas," Journal of Physics D: Applied Physics, vol. 50, 2017.

4 Niemi, K., Schulz-von der Gathen, V., and Dobele, H. F., "Absolute calibration of atomic density measurements by laser-induced fluorescence spectroscopy with two-photon excitation," Journal of Physics D: Applied Physics, vol. 34, 2001, pp. 2330-2335.

Eckbreth, A. C., Laser Diagnostics For Combustion Temperature And Species, CRC Press, 1996.

Loudon, R., The Quantum Theory of Light, Oxford Universty Press, 2000.

Niemi, K., Gathen, V. S. der, and Döbele, H. F., "Absolute atomic oxygen density measurements by twophoton absorption laser-induced fluorescence spectroscopy in an RF-excited atmospheric pressure plasma jet," Plasma Sources Science and Technology, vol. 14, 2005, pp. 375-386.

Brown, M. S., and Jeffries, J. B., "Measurement of atomic concentrations in reacting flows through the use of stimulated gain or loss," Applied Optics, vol. 34, 1995, pp. 1127-1132.

Stancu, G. D., "Laser based spectroscopic diagnostics from UV to Mid-IR applied to the study of atmospheric pressure discharges," Spectroscopy and Spectroscopic: measurement techniques for aerospace flows, D. Giordano and Y. Babou, eds., Von Karman Institute, 2014.

Siegman, A., "Defining, measuring, and optimizing laser beam quality," Proc. SPIE, vol. V, 1993, pp. 2-12.

Kramida, A., Ralchenko, Yu., Reader, J., "NIST Atomic Spectra Database," National Institute of Standards and Technology Available: http://physics.nist.gov/asd.

Dumitrache, C., Butte, C., Eickelberg, A., and Yalin, A. P., "On the Use of REMPI Pre-Ionization for Laser Plasma Formation,” 2018 AIAA Aerospace Sciences Meeting, 2018.

Popov, N. A., "Dissociation of nitrogen in a pulse-periodic dielectric barrier discharge at atmospheric pressure," Plasma Physics Reports, vol. 39, 2013, pp. 420-424.

Levaton, J., Amorim, J., Souza, a R., Franco, D., and Ricard, a, "Kinetics of atoms, metastable, radiative and ionic species in the nitrogen pink afterglow," Journal of Physics D: Applied Physics, vol. 35, 2002, pp. 689699. 
Amorim, J., "Lewis - Rayleigh and Pink Afterglow," IEEE Transactions on Plasma Science, vol. 33, 2005, pp. 368-369.

Volynets, A. V, Lopaev, D. V, Chukalovsky, A. A., Mankelevich, Y., and Popov, N. A., "N 2 dissociation and kinetics of $\mathrm{N}(4 \mathrm{~S})$ atoms in nitrogen DC glow discharge Alternative approaches for ultra low-k materials integration View project Advanced Interconnects View project," Article in Journal of Physics D Applied Physics, vol. 51, Sep. 2018, p. 364002. 